\title{
Theoretical Insight into Organic Dyes Incorporating Triphenylamine-Based Donors and Binary $\pi$-Conjugated Bridges for Dye-Sensitized Solar Cells
}

\author{
Shuxian Wei, ${ }^{1}$ Xiaoqing Lu, ${ }^{1}$ Xiaofan Shi, ${ }^{1}$ Zhigang Deng, ${ }^{1}$ Yang Shao, ${ }^{1}$ Lianming Zhao, \\ Wenyue Guo, ${ }^{1}$ and Chi-Man Lawrence $\mathrm{Wu}^{2}$ \\ ${ }^{1}$ College of Science, China University of Petroleum, Qingdao, Shandong 266580, China \\ ${ }^{2}$ Department of Physics and Materials Science, City University of Hong Kong, Kowloon, Hong Kong
}

Correspondence should be addressed to Xiaoqing Lu; luxq@upc.edu.cn and Wenyue Guo; wyguo@upc.edu.cn

Received 8 April 2014; Revised 11 May 2014; Accepted 19 May 2014; Published 11 June 2014

Academic Editor: Yen-Lin Chen

Copyright (C) 2014 Shuxian Wei et al. This is an open access article distributed under the Creative Commons Attribution License, which permits unrestricted use, distribution, and reproduction in any medium, provided the original work is properly cited.

The design of light-absorbent sensitizers with sustainable and environment-friendly material is one of the key issues for the future development of dye-sensitized solar cells (DSSCs). In this work, a series of organic sensitizers incorporating alkoxy-substituted triphenylamine (tpa) donors and binary $\pi$-conjugated bridges were investigated using density functional theory (DFT) and timedependent DFT (TD-DFT). Molecular geometry, electronic structure, and optical absorption spectra are analyzed in the gas phase, chloroform, and dimethylformamide (DMF) solutions. Our results show that properly choosing the heteroaromatic atoms and/or adding one more alkoxy-substituted tpa group can finely adjust the molecular orbital energy. The solvent effect renders the HOMO-LUMO gaps of the tpa-based sensitizers decrease in the sequence of DMF solution $<$ chloroform solution $<$ gas phase. The absorption spectra are assigned to the ligand-to-ligand charge transfer (LLCT) characteristics via transitions mainly from tpa, 3,4-ethylenedioxythiophene (edot), and alkyl-substituted dithienosilole (dts) groups to edot, dts, and cyanoacrylic acid groups. The binary $\pi$-conjugated bridges play different roles in balancing the electron transfer and recombination for the different tpa-based sensitizers. The protonation/deprotonation effect has great effect on the HOMO-LUMO gaps and thus has great influence on the bands at the long wavelength region, but little influence on the bands at the short wavelength region.

\section{Introduction}

Dye-sensitized solar cells (DSSCs) are currently under active investigation for the solar energy utilization along with the growing worldwide demand for environmentally friendly energy sources [1-7]. The relative high performance, simple fabrication process, and low production costs render DSSCs as competitive alternative to conventional silicon devices. Usually, DSSCs are fabricated by sensitizers (dyes), photoanodes (mesoporous $\mathrm{TiO}_{2}$ films), counterelectrodes, and electrolytes/hole transporters. So far, polypyridyl Ru(II)based complexes are proven to be the most efficient sensitizers employed in DSSCs [8-11], such as the tetraprotonated $\mathrm{Ru}\left(4,4^{\prime}\right.$-dicarboxy-2,2' -bipyridine) ${ }_{2}(\mathrm{NCS})_{2}$ complex (coded as N3) [8] and its doubly protonated analog (coded as N719)
[10]. Although highly efficient, with a high efficiency over $12.1 \%$ [12], Ru-based prototypes are facing the problem of costly synthesis and undesired environmental issues. Metalfree organic sensitizers, which are in good agreement with the trend of the future developments of DSSCs using environmentally friendly and inexpensive materials, show several advantageous features relative to Ru-based complexes: (1) diversity of organic molecular structures can ensure more diversity of sensitizers for DSSCs; (2) high molar extinction coefficients of organic sensitizers can render the use of thinner semiconductor to promote charge separation; (3) more flexibility of organic sensitizers can be beneficial for constructing semitransparent and/or multicolor solar cells. At present, the highest photon-to-electron energy conversion 
efficiency has exceeded $13 \%$ for DSSCs using organic sensitizer [13].

From a theoretical point of view, a few criteria should be fulfilled for the design of ideal organic sensitizers: (1) the molecular orientation should facilitate intramolecular charge transfer, and spatial separation should maintain the photooxidized donor at a distance from the photoinjected electrons, diminishing the impact of back electron transfer processes; (2) the energy level of LUMO should be sufficiently high for efficient electron injection into the $\mathrm{TiO}_{2}$, whereas the energy level of HOMO should be sufficiently low for efficient regeneration of the oxidized state; (3) the absorption band should extend from the whole visible region to near infrared range with strong absorption strength; (4) the stability should be good enough for about $10^{8}$ turnover cycles of exposure to nature $[14,15]$. Based on these requirements, the organic sensitizers are usually designed to be of electron donorconjugated bridge-electron acceptor structure, called $\mathrm{D}-\pi-\mathrm{A}$ architecture, with $\mathrm{TiO}_{2}$ surface anchoring groups integrated into the acceptor moiety. Arylamines and alkylamines, due to strong electron donating abilities as well as efficient intramolecular charge transfer characteristics, are usually used as electron donors in the D- $\pi$-A organic sensitizers [16-23]. Among them, DSSC using this metal-free organic sensitizer incorporating the lipophilic triphenylamine as electron donor and the hydrophilic cyanoacrylic acid as electron acceptor (coded as C219) has achieved an efficiency over $10 \%$ [24].

However, a detailed atomistic characterization of this sensitizer is still not clear enough. Such an atomistic characterization can not only provide information that complements the experimental work, but also help to understand the structural and the electronic properties for design of novel sensitizers with improved performances [25-28]. In this work, we present a theoretical characterization of C219 and its derivatives 1-5. Molecular geometry, electronic structure, and spectral property of the sensitizers are investigated in the gas phase, chloroform, and dimethylformamide (DMF) solutions by means of the density functional theory (DFT) and time-dependent DFT (TD-DFT) calculations.

\section{Computational Methods}

All calculations on the tpa-based sensitizers were performed with DFT and TD-DFT in the Gaussian 09 program package [29]. The B3LYP exchange correlation functional in conjunction with the $6-31 \mathrm{G}(\mathrm{d})$ basis set, which has already been proved to be an optimal compromise between accuracy and computational cost for such large aromatic molecules [30], was employed in the geometrical optimizations without any symmetry constraints for the sensitizers considered in both gas phase and solutions. The solvent effects were evaluated using the nonequilibrium [31] implementation of the conductor-like polarizable continuum model (C-PCM) [32-34]. This approach provides results very close to those obtained by the original dielectric model for high dielectric constant solvents but is significantly more effective in geometry optimizations and less prone to the numerical errors arising from the small part of the solute electron cloud lying outside the cavity [34]. TD-DFT calculations were used to investigate the optical properties of the sensitizers using the approximation of $E_{0-0}$ with the lowest vertical excitation energy of the system at the ground state geometry, which can be accurately and efficiently calculated by TD-DFT [35]. The 50 lowest spin-allowed singlet-singlet transitions, up to an energy of at least $\sim 4.0 \mathrm{eV}$, were taken into account in the calculations of the adsorption spectra.

\section{Results and Discussion}

In the following sections, we start with the geometrical description of C219 and its derivatives, followed by the discussion of electronic structures and molecular orbital energy levels and then the analysis of absorption spectra in the real environment of chloroform and DMF solutions, and finally the comparison of the relative light-harvesting efficiency (RLHE) of the tpa-based sensitizers.

3.1. Geometrical Considerations. The chemical structures of C219 and its derivatives 1-5 are shown in Figure 1. C219 is composed of three parts: (1) an alkoxy-substituted triphenylamine (tpa) as donor, (2) a cyanoacrylic acid as acceptor and anchoring group, and (3) a binary $\pi$-conjugated unit consisting of 3,4-ethylenedioxythiophene (edot) and alkylsubstituted dithienosilole (dts) as bridge [24]. For computational convenience, the $\mathrm{C}_{6} \mathrm{H}_{13}{ }^{+}$and $\mathrm{C}_{8} \mathrm{H}_{17}{ }^{+}$groups of the experimental $\mathbf{C 2 1 9}$ sensitizer are replaced by methyl groups marked with blue dash-lined ellipses and red dash-lined circles, as shown in Figure 1. Considering that the electron transfer mainly takes place via the high $\pi$-electron conjugated tpa center to acceptor and/or bridge centers, the end-on substitutions of the $\mathrm{C}_{6} \mathrm{H}_{13}{ }^{+}$and $\mathrm{C}_{8} \mathrm{H}_{17}{ }^{+}$groups by methyl groups have slight influence on the spectral properties of the C219 sensitizer.

In fact, these substitutions are checked in order not to result in appreciable changes in the electronic structures as well as the absorption spectra. The $\mathrm{S}$ atoms in the conjugated bridge of the prototype molecule $\mathrm{C} 219$ are substituted by the same electron-rich heteroaromatic $\mathrm{O}$ and $\mathrm{Se}$ atoms in sensitizers $\mathbf{1}$ and $\mathbf{2}$. For sensitizers $\mathbf{3}, \mathbf{4}$, and $\mathbf{5}$, one more alkoxysubstituted tpa group is added to substitute the ethylenedioxy group in 1, C219, and 2. This structural design is based on two considerations: (1) the heteroaromatic atoms may adjust the molecular orbital energies appropriately and (2) one more alkoxy-substituted tpa group may not only enhance the electron-donating ability, but also effectively inhibit $\mathrm{I}_{3}{ }^{-}$ from approaching the surface of $\mathrm{TiO}_{2}$ by forming a denser layer at the surface. In addition, $\mathrm{Na}^{+}$is used to substitute for $\mathrm{H}^{+}$on cyanoacrylic acid groups in order to evaluate the protonation/deprotonation effect, that is, $\mathbf{1}^{\prime}, \mathbf{C} 219^{\prime}, \mathbf{2}^{\prime}, \mathbf{3}^{\prime}, \mathbf{4}^{\prime}$, and $\mathbf{5}^{\prime}$, as shown in Figure 1.

According to the experimental setup of $\mathbf{C 2 1 9}$, the solvents for calculations are chloroform (relative dielectric constant EPS $=4.71)$ and DMF $(E P S=37.22)$. The spectra recorded in different solvents are available, thus rendering it conceivable to investigate the solvent effects. For all the ground state 


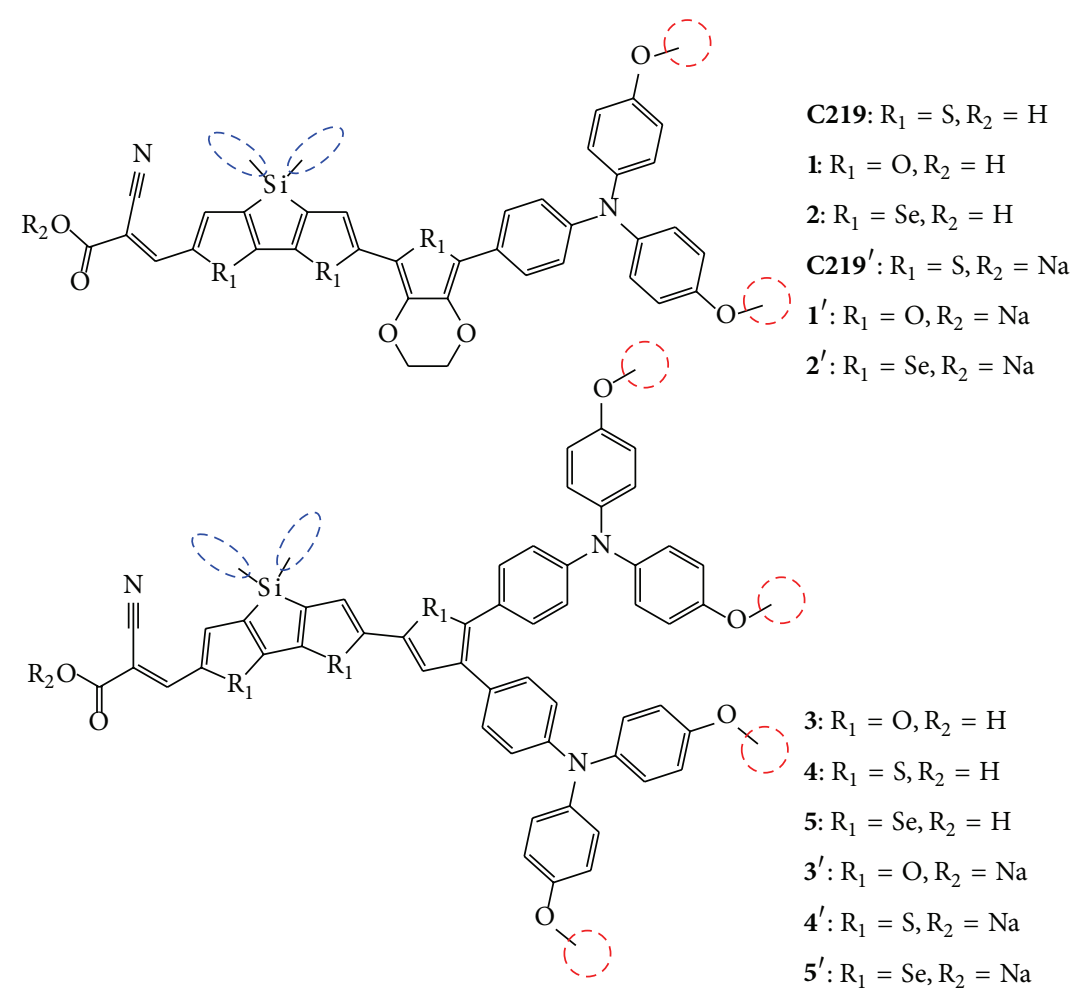

Figure 1: Schematic structures of the tpa-based sensitizers. The $\mathrm{C}_{6} \mathrm{H}_{13}{ }^{+}$and $\mathrm{C}_{8} \mathrm{H}_{17}{ }^{+}$groups in the tpa-based sensitizers are replaced by methyl groups marked with blue dash-lined ellipses and red dash-lined circles.

geometries, our results show that different solvents have no noticeable impact because of the difference of the solvent polarity.

3.2. The Frontier Orbitals and Energy Levels. The electronic excitation occurs mainly from the highest occupied molecular orbitals (HOMOs) to the lowest unoccupied molecular orbitals (LUMOs), and usually the HOMOs localize on the donor subunit and LUMOs on the acceptor subunit. The electronic structure analysis is thus essential to evaluate whether the molecular orbital contributions facilitate the efficient charge separation or not. Considering the similarity of their geometries, $\mathbf{C 2 1 9}$ and $\mathbf{C 2 1 9 ^ { \prime }}$ are chosen to exhibit the frontier molecular orbitals for 1, C219, 2 and $1^{\prime}, \mathrm{C} 219^{\prime}, 2^{\prime}, 4$ and $\mathbf{4}^{\prime}$ are used to show the frontier molecular orbitals for 3 5 and $3^{\prime}-5^{\prime}$, respectively. Figure 2 shows the selected frontier

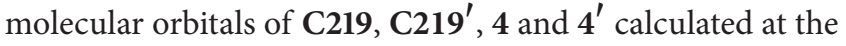
B3LYP/6-31G(d) level in chloroform solution. The first six frontier molecular orbital energies and the HOMO-LUMO gaps are depicted in Figure 3.

It is clear, seen from Figure 2, that the first three HOMOs (HOMO, HOMO-1, and HOMO-2) of C219 are mainly composed of the $\pi$ combinations of $\mathrm{C}$ and $\mathrm{N}$ on tpa, edot, and dts groups. Particularly, for the HOMO, considerable contribution is from the tpa ligand. This point is confirmed as one more tpa ligand is introduced in $\mathbf{4}$, in which the first two HOMOs show almost entirely tpa-based characteristics.
The bridge involved or not in the HOMOs would be of great difference for the electron transfer. If the binary bridge of edot and dts is involved in the HOMOs, it would facilitate the direct transfer of electron from the donor to the acceptor subunit, but it is also easy to occur recombinations for the transferred electrons. Otherwise, it would prevent electron recombination effectively but is not beneficial for the electron transfer. The key point for the binary bridge is that spatial separation should balance the photooxidized donor at a distance from the photoinjected electrons, diminishing the impact of back electron transfer processes [24, 36-39]. In this sense, the binary bridge of edot and dts in C219 plays a more positive role in promoting electron transfer but a more negative role in diminishing back electron transfer than that in 4 [24]. That is, the binary $\pi$-conjugated bridges play different roles in balancing the electron transfer and recombination for the different tpa-based sensitizers.

The first three LUMOs of $\mathbf{C 2 1 9}$ are delocalized through the edot, dts, and cyanoacrylic acid groups, as shown in Figure 2. Sizeable contributions are from the cyanoacrylic acid group, which would facilitate the excited electrons injection into the semiconductors directly. The $\pi^{*}$ orbitals from tpa ligand are almost not involved in the LUMOs. Considering that the LUMOs also include the spatial separation, it would be favorable for the electron recombination if the binary bridge of edot and dts is involved in the HOMOs. That is, 4 and $4^{\prime}$ are much better than C219 and C219' for 
C219
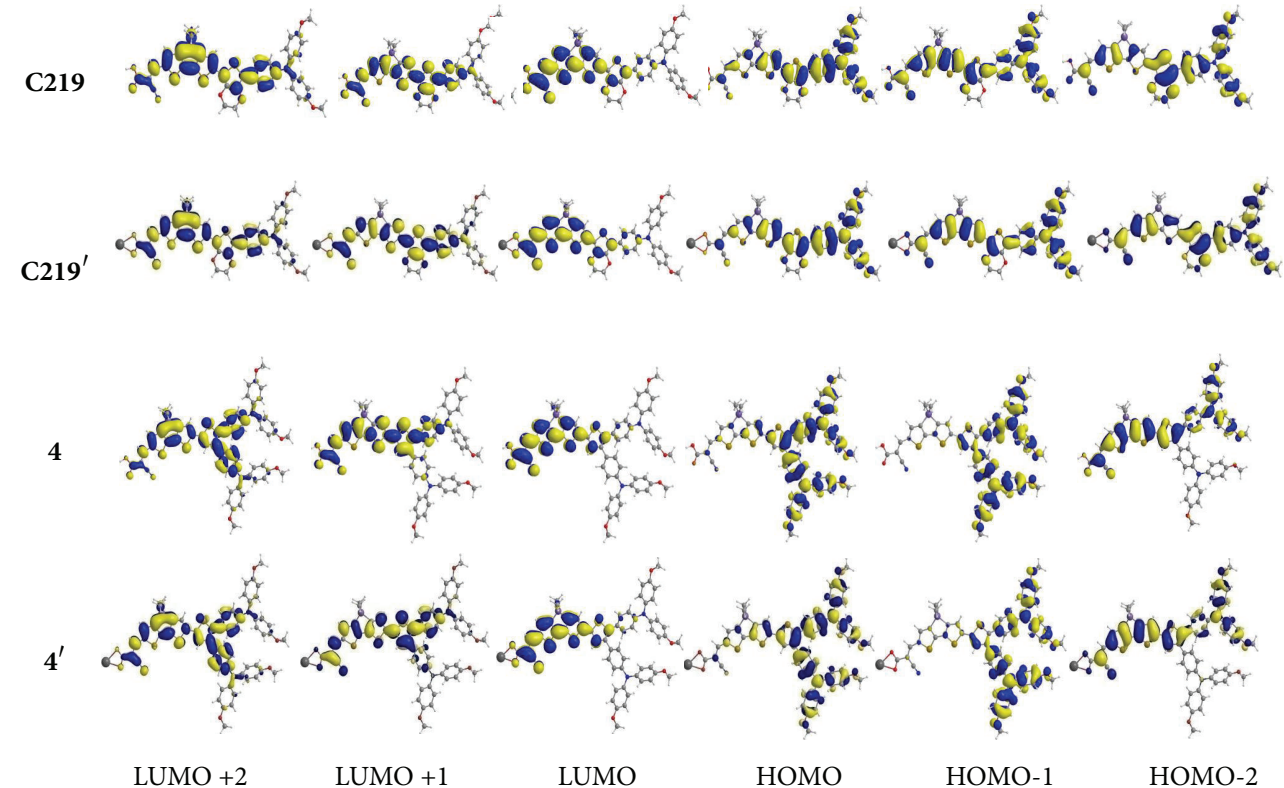

FIgURE 2: The frontier molecular orbitals of sensitizers C219, C219', 4 , and $4^{\prime}$. Isodensity contour $=0.02$.

diminishing the impact of back electron transfer. Seen from Figure 2, substitutions of $\mathrm{Na}^{+}$for $\mathrm{H}^{+}\left(\mathbf{C} 219,4 \rightarrow \mathrm{C}^{219}{ }^{\prime}, 4^{\prime}\right)$ on cyanoacrylic acid groups have more effects on the LUMOs than the HOMOs, as mirrored by the alterations of the $\mathrm{Na}^{+}$associated cyanoacrylic acid orbital shapes. Substitutions of $\mathrm{O}$ and Se for $\mathrm{S}$ atom on the bridge alter the energy levels of the frontier molecular orbitals obviously. The energy levels of both HOMO and LUMO in the same framework are in the sequence of $5<\mathbf{4}<3$ and $2<\mathrm{C} 219<\mathbf{1}$ in the gas phase and solutions, as shown in Figure 3. This is due to the electron donating ability of the heteroatom involved, $\mathrm{Se}>\mathrm{S}$ $>\mathrm{O}$, which stabilizes the complexes by lowering their energy levels. Similar trend appears for the $\mathrm{Na}^{+}$-substituted analogs.

As seen from Figure 3, the HOMO-LUMO gaps of the tpa-based sensitizers decrease in the sequence of DMF solution < chloroform solution < gas phase. For instance, the HOMO-LUMO gaps of $\mathbf{C 2 1 9}$ are 1.92, 1.97, and $2.09 \mathrm{eV}$ obtained in DMF, chloroform, and the gas phase, respectively. This trend is due to the interactions of the sensitizers and the surroundings with different relative dielectric constant. As the relative dielectric constant increases, that is, gas phase $(\mathrm{EPS}=0.00)<$ chloroform solution $(\mathrm{EPS}=4.71)<\mathrm{DMF}$ solution $(\mathrm{EPS}=37.22$ ), the interaction between the tpabased sensitizers and the surroundings increases, and thus intramolecular interactions become weak and their energy levels increase $[40,41]$. The tpa-based sensitizers are less insensitive to the change of solvent, as mirrored by a $0.05 \mathrm{eV}$ shift when going from chloroform solution to DMF solution, while more sensitive to the change when going from the gas phase to the solutions. Besides, substitutions of $\mathrm{Na}^{+}$ for $\mathrm{H}^{+}$on cyanoacrylic acid groups have great effect on the HOMO-LUMO gaps. As shown in Figure 3, the values increase by 0.28 and $0.31 \mathrm{eV}$ when going from $\mathrm{C} 219$ and 4 to C219' and $\mathbf{4}^{\prime}$, respectively. This is ascribed to the fact that these substitutions destabilize the LUMOs greater than the HOMOs and thus enlarge the HOMO-LUMO gaps, in good consistency with the alteration trend of the frontier molecular orbital distributions.

3.3. Electronic Excitations and Absorption Spectra. The absorption spectra of the tpa-based sensitizers are shown in Figure 4, in which $\mathrm{H}^{+}$- and $\mathrm{Na}^{+}$-contained sensitizers are depicted in Figures 4(a) and 4(b), respectively. Overall, the band line shapes obtained in the gas phase and solutions agree well with each other, but the spectra obtained in solutions are shifted toward the long wavelength region with respect to those obtained in the gas phase $[42,43]$. Comparison of the results gained in two solutions shows that the spectra in DMF solution are slightly red-shifted with respect to those in chloroform solution. This trend is in good agreement with the sequence of the HOMO-LUMO gaps obtained in two solutions. As for the protonation/deprotonation effect, substitutions of $\mathrm{Na}^{+}$for $\mathrm{H}^{+}$render the spectra toward the short wavelength region, as shown in Figures 4(a) and 4(b). This trend agrees well with the enlarged HOMO-LUMO gaps of tpa-based sensitizers caused by these substitutions. In view of the real environment of DSSCs and the experimental setups, the results obtained in chloroform solution are used for the discussion of spectral properties as follows. Table 1 lists the selected excitation energies $(E, \mathrm{~nm})$, oscillator strength $(f)$, and relative orbital contributions to the optical transitions between 350 and $800 \mathrm{~nm}$ of the absorption spectra in chloroform solution. 


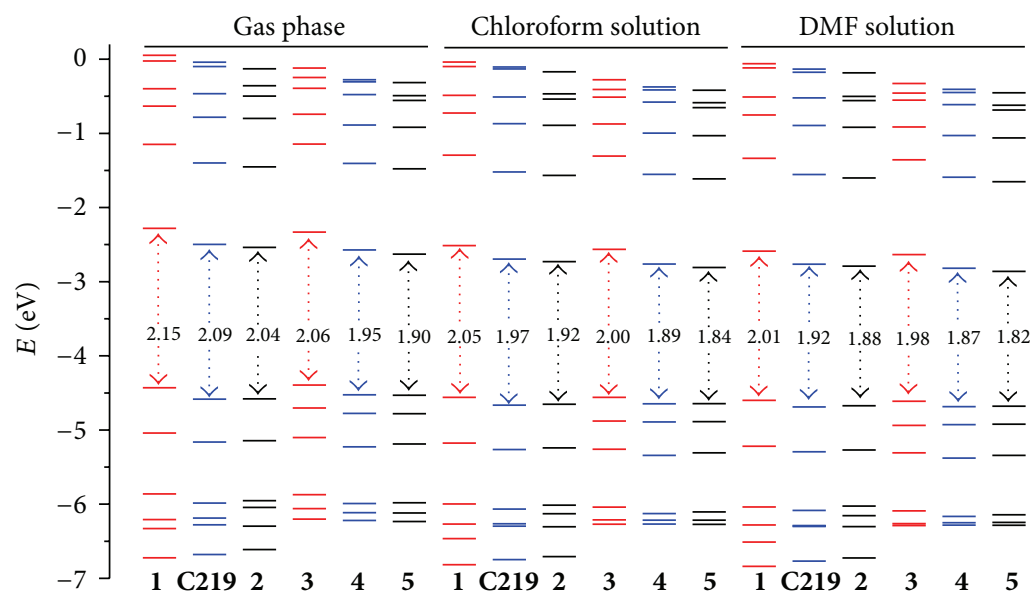

(a)

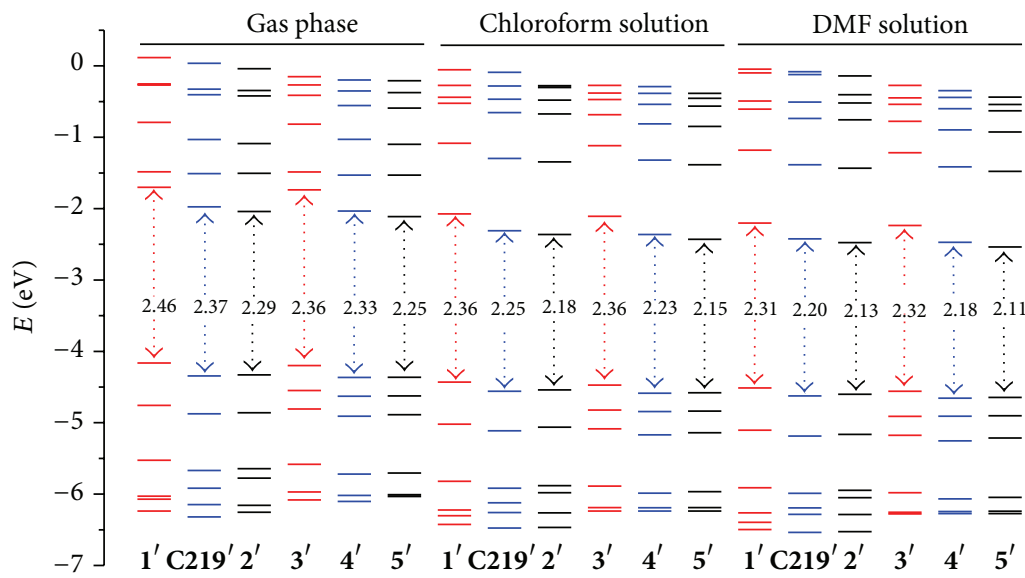

(b)

FIGURE 3: Energy levels and HOMO-LUMO gaps of the tpa-based sensitizers.

For C219 in chloroform solution, four separated absorption bands are found to center at $\sim 360, \sim 439, \sim 529$, and $\sim$ $714 \mathrm{~nm}$. At $\sim 360 \mathrm{~nm}$, the band is composed of two peaks at 356.4 and $363.3 \mathrm{~nm}$, originated from the starting orbitals of the HOMO/HOMO-1 combinations to the arriving orbitals of LUMO+1/LUMO+2 (see Table 1). Frontier orbital analysis shows that this band has the interligand and intraligand ligand-to-ligand charge transfer (LLCT) characteristics; that is, transitions occur from tpa, edot, and dts groups to edot, dts, and cyanoacrylic acid groups. Similarly, the bands at $\sim 439, \sim 529$, and $\sim 714 \mathrm{~nm}$ also show interligand LLCT characteristics. It is quite different from the metal-centered complexes in previous investigations [25-28], in which spectra at the short wavelengths of the visible region show mainly the LLCT characteristic while those at the long wavelengths of the visible region show mixed characteristics of metal-toligand charge transfer (MLCT) and LLCT. For C219, there is an increasing trend of oscillator strength (which is regarded as the measure of the intensity of the absorption peaks [37, 44]) along with the shift of the absorption band toward the long wavelength region; that is, the stronger oscillator strength appears in the band at the longer wavelength region. The lowest vertical excitation for C219 occurs at $713.1 \mathrm{~nm}$ with the oscillator strength of 1.281 , originated from the $100 \%$ HOMO $\rightarrow$ LUMO transition. This transition occurs from tpa, edot, and dts groups to edot, dts, and cyanoacrylic acid groups, a mixed LLCT characteristic. Similar spectra and electronic excitation trends also appear for the O- and Sesubstituted sensitizers 1 and 2, as shown in Figure 4(a).

The spectra tend to shift toward the short wavelength region when substituting $\mathrm{Na}^{+}$for $\mathrm{H}^{+}$, as shown in Figure 4(b). For $\mathrm{C}^{219}{ }^{\prime}$ in chloroform solution, four separated absorption bands are found to center at $\sim 350, \sim 424, \sim 488$, and $\sim 630 \mathrm{~nm}$. Compared with the corresponding bands of C219, the substitution of $\mathrm{Na}^{+}$for $\mathrm{H}^{+}$has little effect on the bands at the short wavelength region $(\sim 360$ and $\sim 439 \mathrm{~nm}$ versus $\sim 350$ and $\sim 424 \mathrm{~nm}$ ) but has great effect on the bands at the long wavelength region ( $\sim 529$ and $\sim 714$ nm versus $\sim 488$ and $\sim 630 \mathrm{~nm})$. However, the increasing trend of oscillator strength in $\mathbf{1}^{\prime}, \mathbf{C 2 1 9 ^ { \prime }}$ and $\mathbf{2}^{\prime}$ is still the same as that in C219 along with the absorption band toward the long 

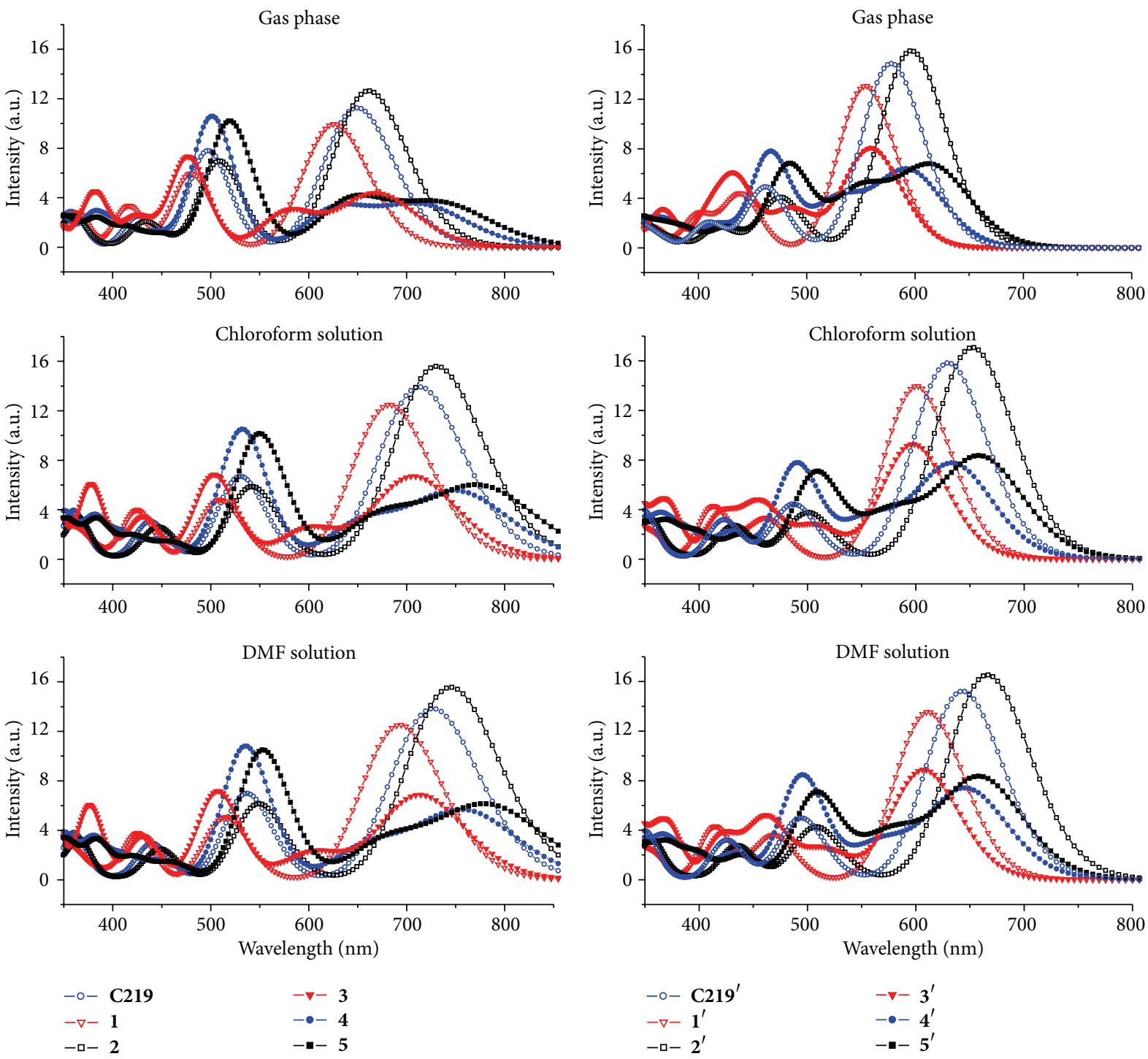

(a)

(b)

FiguRE 4: Simulated absorption spectra of $\mathrm{H}^{+}-$(a) and $\mathrm{Na}^{+}$- (b) contained tpa-based sensitizers.

wavelength region. The lowest vertical excitation for $\mathbf{C 2 1 9}$ occurs at $630.0 \mathrm{~nm}$ with the stronger oscillator strength of 1.456 than the corresponding value of C219. In the energy range investigated, the band calculated at $\sim 630 \mathrm{~nm}$ for $\mathbf{C 2 1 9 ^ { \prime }}$ is in good agreement with the experimental result of $584 \mathrm{~nm}$, more consistent than $\mathbf{C 2 1 9}$ at $714 \mathrm{~nm}$ [24]. This means the spectra of the tpa-based organic sensitizers, as well as the energy levels and the HOMO-LUMO gaps, are sensitive to the protonation/deprotonation effect.

The introduction of one more alkoxy-substituted tpa group alters the spectra significantly, as shown in Figure 4. For 4 in chloroform solution, the oscillator strength of bands becomes weaker at the long wavelength region of $600-800 \mathrm{~nm}$, while stronger at middle wavelength region of $500-600 \mathrm{~nm}$ than the corresponding values of C219.
With respect to oscillator strength, it will be affected by the electron-donating capability from $\pi$-conjugation and heteroaromatic groups in sensitizers [27, 44, 45]. Similar trends occur for the $\mathrm{O}$ - and Se-substituted sensitizers 3, 5, and their $\mathrm{Na}^{+}$-substituted sensitizers $3^{\prime}, 4^{\prime}$, and $5^{\prime}$. For 4 at $\sim 532 \mathrm{~nm}$, the band results from the $98 \%$ HOMO- $2 \rightarrow$ LUMO transition with the oscillator strength of 0.967 (see Table 1). This transition shows the interligand and intraligand LLCT characteristic from tpa, edot, dts, and even cyanoacrylic acid groups to edot, dts, and cyanoacrylic acid groups. The lowest vertical excitation for 4 occurs at $754.3 \mathrm{~nm}$ with the oscillator strength of 0.487 , originated from the $100 \% \mathrm{HOMO} \rightarrow$ LUMO transition. Obviously, this transition has interligand LLCT characteristic; that is, transition occurs from double tpa groups to edot, dts, and cyanoacrylic acid groups. 
TABLE 1: Selected excitation energies $(E, \mathrm{~nm})$, oscillator strength $(f)$, and relative orbital contributions to the optical transitions between 350 and $800 \mathrm{~nm}$ of the absorption spectra of tpa-based sensitizers in chloroform solution ${ }^{\mathrm{a}}$.

\begin{tabular}{|c|c|c|c|c|c|}
\hline$E$ & $f$ & Composition & $E$ & $f$ & Composition \\
\hline \multicolumn{3}{|c|}{ C219 } & \multicolumn{3}{|c|}{$\mathrm{C} 219^{\prime}$} \\
\hline 713.1 & 1.281 & $\mathrm{H}-0 \rightarrow \mathrm{L}+0(100 \%)$ & 630.0 & 1.456 & $\mathrm{H}-0 \rightarrow \mathrm{L}+0(99 \%)$ \\
\hline 530.4 & 0.616 & $\mathrm{H}-1 \rightarrow \mathrm{L}+0(97 \%)$ & 487.4 & 0.409 & $\mathrm{H}-1 \rightarrow \mathrm{L}+0(97 \%)$ \\
\hline 438.5 & 0.281 & $\mathrm{H}-0 \rightarrow \mathrm{L}+1(88 \%)$ & 423.0 & 0.292 & $\mathrm{H}-0 \rightarrow \mathrm{L}+1(91 \%)$ \\
\hline 387.3 & 0.023 & $\begin{array}{l}\mathrm{H}-3 \rightarrow \mathrm{L}+0(72 \%) \\
\mathrm{H}-1 \rightarrow \mathrm{L}+1(14 \%)\end{array}$ & 354.5 & 0.085 & $\begin{array}{l}\mathrm{H}-0 \rightarrow \mathrm{L}+2(67 \%) \\
\mathrm{H}-1 \rightarrow \mathrm{L}+1(24 \%)\end{array}$ \\
\hline 363.3 & 0.212 & $\mathrm{H}-0 \rightarrow \mathrm{L}+2(86 \%)$ & 350.5 & 0.023 & $\mathrm{H}-0 \rightarrow \mathrm{L}+3(90 \%)$ \\
\hline 356.4 & 0.152 & $\mathrm{H}-1 \rightarrow \mathrm{L}+1(68 \%)$ & & & \\
\hline \multicolumn{3}{|r|}{ 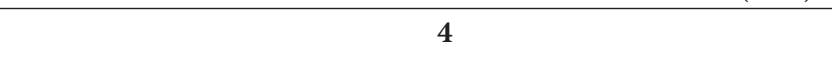 } & \multicolumn{3}{|c|}{$4^{\prime}$} \\
\hline 754.3 & 0.487 & $\mathrm{H}-0 \rightarrow \mathrm{L}+0(100 \%)$ & 635.1 & 0.699 & $\mathrm{H}-0 \rightarrow \mathrm{L}+0(99 \%)$ \\
\hline 658.2 & 0.286 & $\mathrm{H}-1 \rightarrow \mathrm{L}+0(99 \%)$ & 559.7 & 0.314 & $\mathrm{H}-1 \rightarrow \mathrm{L}+0(98 \%)$ \\
\hline 532.3 & 0.967 & $\mathrm{H}-2 \rightarrow \mathrm{L}+0(98 \%)$ & 490.5 & 0.713 & $\mathrm{H}-2 \rightarrow \mathrm{L}+0(97 \%)$ \\
\hline 450.5 & 0.137 & $\mathrm{H}-0 \rightarrow \mathrm{L}+1(94 \%)$ & 429.5 & 0.201 & $\mathrm{H}-0 \rightarrow \mathrm{L}+1(93 \%)$ \\
\hline 412.3 & 0.170 & $\begin{array}{l}\mathrm{H}-1 \rightarrow \mathrm{L}+1(79 \%) \\
\mathrm{H}-3 \rightarrow \mathrm{L}+0(17 \%)\end{array}$ & 390.6 & 0.193 & $\mathrm{H}-1 \rightarrow \mathrm{L}+1(88 \%)$ \\
\hline 382.5 & 0.078 & $\begin{array}{l}\mathrm{H}-4 \rightarrow \mathrm{L}+0(69 \%) \\
\mathrm{H}-0 \rightarrow \mathrm{L}+2(25 \%)\end{array}$ & 366.4 & 0.267 & $\mathrm{H}-0 \rightarrow \mathrm{L}+2(81 \%)$ \\
\hline 381.9 & 0.211 & $\begin{array}{l}\mathrm{H}-0 \rightarrow \mathrm{L}+2(62 \%) \\
\mathrm{H}-4 \rightarrow \mathrm{L}+0(30 \%)\end{array}$ & 353.6 & 0.029 & $\mathrm{H}-0 \rightarrow \mathrm{L}+3(88 \%)$ \\
\hline 355.6 & 0.109 & $\begin{array}{l}\mathrm{H}-2 \rightarrow \mathrm{L}+1(59 \%) \\
\mathrm{H}-6 \rightarrow \mathrm{L}+0(24 \%)\end{array}$ & 350.9 & 0.076 & $\mathrm{H}-2 \rightarrow \mathrm{L}+1(70 \%)$ \\
\hline 352.2 & 0.029 & $\mathrm{H}-0 \rightarrow \mathrm{L}+3(88 \%)$ & & & \\
\hline 349.1 & 0.201 & $\mathrm{H}-1 \rightarrow \mathrm{L}+2(90 \%)$ & & & \\
\hline
\end{tabular}

${ }^{a}$ Only oscillator strength $f>0.02$ and orbital percentage $>10 \%$ are reported, where $\mathrm{H}=\mathrm{HOMO}$ and $\mathrm{L}=\mathrm{LUMO}$.

TABLE 2: The HOMO and LUMO energies, HOMO-LUMO gaps, lowest vertical excitation energies $\left(E_{A}\right)$, oscillator strengths $(f)$, and relative LHE (RLHE) for all sensitizers ${ }^{\mathrm{a}}$.

\begin{tabular}{|c|c|c|c|c|c|c|c|}
\hline Solutions & Sensitizers & LUMO & HOMO & HOMO-LUMO & $E_{A}$ & $f$ & RLHE \\
\hline \multirow{6}{*}{ Chloroform } & 1 & -2.51 & -4.56 & 2.05 & 1.82 & 1.150 & 0.980 \\
\hline & C219 & -2.70 & -4.67 & 1.97 & 1.74 & 1.281 & 1.000 \\
\hline & 2 & -2.73 & -4.65 & 1.92 & 1.70 & 1.435 & 1.016 \\
\hline & 3 & -2.56 & -4.56 & 2.00 & 1.76 & 0.614 & 1.123 \\
\hline & 4 & -2.76 & -4.65 & 1.89 & 1.65 & 0.487 & 1.000 \\
\hline & 5 & -2.81 & -4.64 & 1.84 & 1.60 & 0.524 & 1.040 \\
\hline \multirow{6}{*}{ DMF } & 1 & -2.59 & -4.60 & 2.01 & 1.79 & 1.153 & 0.982 \\
\hline & C219 & -2.76 & -4.69 & 1.92 & 1.71 & 1.273 & 1.000 \\
\hline & 2 & -2.79 & -4.67 & 1.88 & 1.67 & 1.431 & 1.017 \\
\hline & 3 & -2.64 & -4.61 & 1.98 & 1.74 & 0.630 & 1.120 \\
\hline & 4 & -2.82 & -4.69 & 1.87 & 1.63 & 0.500 & 1.000 \\
\hline & 5 & -2.86 & -4.68 & 1.82 & 1.58 & 0.537 & 1.038 \\
\hline
\end{tabular}

${ }^{\mathrm{a}}$ All energies are in $\mathrm{eV}$.

3.4. Light-Harvesting Efficiency (LHE). Light-harvesting efficiency (LHE), as a good indicator of the incident photon-toelectron conversion efficiency (IPCE), characterizes the ability of dyes in harvesting light. The LHE can be approximately expressed as [46]

$$
\mathrm{LHE}=1-10^{-A}=1-10^{-f}
$$

where $A(f)$ is the absorption (oscillator strength) of the dye associated with the $\lambda_{\text {max }}$, that is, the lowest vertical excitation energy $\left(E_{\mathrm{A}}\right)$. Table 2 lists the HOMO and LUMO energies, HOMO-LUMO gaps, lowest vertical excitation energies $\left(E_{A}\right)$, oscillator strengths $(f)$, and relative LHE (RLHE) of all sensitizers calculated in chloroform and DMF solutions. According to the structural and spectral properties, the relative LHE (RLHE) is evaluated by comparing sensitizers 
1, C219 and 2 with C219, sensitizers 3, 4, and 5 with 4 . The influence of solutions on LHE is negligible, due to the little difference in $E_{\mathrm{A}}$ and $f$ for sensitizers in different solutions, as shown in Table 2. For 1, C219 and $\mathbf{2}$ in chloroform solution, 2 owns the highest RLHE of 1.016, followed by C219 with 1.000 and $\mathbf{1}$ of 0.980 . This trend is in good agreement with the sequence of electron donating ability of heteroatoms involved, $\mathrm{Se}>\mathrm{S}>\mathrm{O}$. For 3, 4, and $\mathbf{5}$ in chloroform solution, 3 shows the highest RLHE of 1.123, followed by $\mathbf{5}$ of 1.040 and 4 of 1.000. This indicates that the adjustment on the LHE via the heteroatoms would not be of much help after greatly enhancing the electron-donating group, such as another tpa donor involved.

\section{Conclusions}

In this work, we have presented a theoretical investigation on organic sensitizers incorporating alkoxy-substituted triphenylamine donors and binary $\pi$-conjugated bridges based on DFT/TD-DFT calculations in the gas phase, chloroform, and DMF solutions. Our results show that properly choosing the heteroaromatic atoms and adding one more alkoxysubstituted tpa group can finely adjust the molecular orbital energies. Frontier orbital analysis shows that the three highest HOMOs are mainly composed of the $\pi$ combinations on tpa, edot, and dts groups for C219-like sensitizers but only tpa group for 4-like analogs. The three LUMOs are delocalized through the edot, dts, and cyanoacrylic acid groups for all tpa-based sensitizers. The HOMO-LUMO gaps of the tpabased sensitizers decrease in the sequence of DMF solution $<$ chloroform solution $<$ gas phase, according well to the increase of relative dielectric constant of gas phase $<$ chloroform solution $<$ DMF solution. The spectra are assigned to the LLCT characteristics which are originated mainly from transitions of tpa, edot, and dts groups to edot, dts, and cyanoacrylic acid groups. The binary $\pi$-conjugated bridge plays a dual role in balancing the electron transfer and recombination for C219-like sensitizers but an active role in diminishing the impact of back electron transfer for 4like analogs. The protonation/deprotonation effect enlarges the HOMO-LUMO gaps and thus has great influence on the bands at the long wavelength region, but little influence on the bands at the short wavelength region.

\section{Conflict of Interests}

The authors declare that there is no conflict of interests regarding the publication of this paper.

\section{Acknowledgments}

This work was supported by NSFC (21303266), Shandong Province Natural Science Foundation (ZR2011EMZ002), Promotive Research Fund for Excellent Young and Middle-Aged Scientists of Shandong Province (BS2013CL031), PetroChina Innovation Foundation (2013D-5006-0406), and the Fundamental Research Funds for the Central Universities (13CX05004A, 13CX05020A, and 13CX02025A).

\section{References}

[1] B. O'Regan and M. Grätzel, "A low-cost, high-efficiency solar cell based on dye-sensitized colloidal $\mathrm{TiO}_{2}$ films," Nature, vol. 353, no. 6346, pp. 737-740, 1991.

[2] A. Hagfeldt and M. Grätzel, "Molecular photovoltaics," Accounts of Chemical Research, vol. 33, no. 5, pp. 269-277, 2000.

[3] M. Grätzel, "Photoelectrochemical cells," Nature, vol. 414, no. 6861, pp. 338-344, 2001.

[4] M. Grätzel, "Dye-sensitized solar cells," Journal of Photochemistry and Photobiology C: Photochemistry Reviews, vol. 4, no. 2, pp. 145-153, 2003.

[5] M. Grätzel, "Applied physics: solar cells to dye for," Nature, vol. 421, no. 6923, pp. 586-587, 2003.

[6] M. Grätzel, "Solar energy conversion by dye-sensitized photovoltaic cells," Inorganic Chemistry, vol. 44, no. 20, pp. 6841-6851, 2005.

[7] L. M. Goncalves, V. de Zea Bermudez, H. A. Ribeiro, and A. M. Mendes, "Dye-sensitized solar cells: a safe bet for the future," Energy \& Environmental Science, vol. 1, no. 6, pp. 655-667, 2008.

[8] M. K. Nazeeruddin, A. Kay, I. Rodicio et al., "Conversion of light to electricity by cis- $\mathrm{X}_{2}$ bis $\left(2,2^{\prime}\right.$-bipyridyl- $4,4^{\prime}$ dicarboxylate)ruthenium(II) charge-transfer sensitizers ( $\mathrm{X}=$ $\mathrm{Cl}-, \mathrm{Br}-, \mathrm{I}-, \mathrm{CN}-$, and $\mathrm{SCN}-$ ) on nanocrystalline $\mathrm{TiO}_{2}$ electrodes," Journal of the American Chemical Society, vol. 115, no. 14, pp. 6382-6390, 1993.

[9] P. Wang, S. M. Zakeeruddin, J. E. Moser et al., "Stable new sensitizer with improved light harvesting for nanocrystalline dye-sensitized solar cells," Advanced Materials, vol. 16, no. 20, pp. 1806-1811, 2004.

[10] M. K. Nazeeruddin, F. de Angelis, S. Fantacci et al., "Combined experimental and DFT-TDDFT computational study of photoelectrochemical cell ruthenium sensitizers," Journal of the American Chemical Society, vol. 127, no. 48, pp. 16835-16847, 2005.

[11] Y. Chiba, A. Islam, Y. Watanabe, R. Komiya, N. Koide, and L. Han, "Dye-sensitized solar cells with conversion efficiency of 11.1\%," Japanese Journal of Applied Physics, vol. 45, no. 25, pp. 638-640, 2006.

[12] Q. J. Yu, Y. H. Wang, Z. H. Yi et al., "High-efficiency dyesensitized solar cells: the influence of lithium ions on exciton dissociation, charge recombination, and surface states," ACS Nano, vol. 4, no. 10, pp. 6032-6038, 2010.

[13] S. Mathew, A. Yella, P. Gao et al., "Dye-sensitized solar cells with $13 \%$ efficiency achieved through the molecular engineering of porphyrin sensitizers," Nature Chemistry, vol. 6, no. 3, pp. 242247, 2014.

[14] M. Grätzel, "Conversion of sunlight to electric power by nanocrystalline dye-sensitized solar cells," Journal of Photochemistry and Photobiology A: Chemistry, vol. 164, no. 1-3, pp. 3-14, 2004.

[15] N. Robertson, "Optimizing dyes for dye-sensitized solar cells," Angewandte Chemie-International Edition, vol. 45, no. 15, pp. 2338-2345, 2006.

[16] T. Horiuchi, H. Miura, K. Sumioka, and S. Uchida, "High efficiency of dye-sensitized solar cells based on metal-free indoline dyes," Journal of the American Chemical Society, vol. 126, no. 39, pp. 12218-12219, 2004.

[17] K. Hara, T. Sato, R. Katoh et al., "Novel conjugated organic dyes for efficient dye-sensitized solar cells," Advanced Functional Materials, vol. 15, no. 2, pp. 246-252, 2005. 
[18] D. P. Hagberg, T. Edvinsson, T. Marinado, G. Boschloo, A. Hagfeldt, and L. Sun, "A novel organic chromophore for dyesensitized nanostructured solar cells," Chemical Communications, no. 21, pp. 2245-2247, 2006.

[19] N. Koumura, Z.-S. Wang, S. Mori, M. Miyashita, E. Suzuki, and K. Hara, "Alkyl-functionalized organic dyes for efficient molecular photovoltaics," Journal of the American Chemical Society, vol. 128, no. 44, pp. 14256-14257, 2006.

[20] S. Kim, J. K. Lee, S. O. Kang et al., "Molecular engineering of organic sensitizers for solar cell applications," Journal of the American Chemical Society, vol. 128, no. 51, pp. 16701-16707, 2006.

[21] H. Choi, J. K. Lee, K. Song, S. O. Kang, and J. Ko, "Novel organic dyes containing bis-dimethylfluorenyl amino benzo[b]thiophene for highly efficient dye-sensitized solar cell," Tetrahedron, vol. 63, no. 15, pp. 3115-3121, 2007.

[22] Z. J. Ning and H. Tian, "Triarylamine: a promising core unit for efficient photovoltaic materials," Chemical Communications, no. 37, pp. 5483-5495, 2009.

[23] Z. J. Ning, Q. Zhang, H. C. Pei et al., "Photovoltage improvement for dye-sensitized solar cells via cone-shaped structural design," The Journal of Physical Chemistry C, vol. 113, no. 23, pp. 1030710313, 2009.

[24] W. D. Zeng, Y. M. Cao, Y. Bai et al., "Efficient dye-sensitized solar cells with an organic photosensitizer featuring orderly conjugated ethylenedioxythiophene and dithienosilole blocks," Chemistry of Materials, vol. 22, no. 5, pp. 1915-1925, 2010.

[25] X. Q. Lu, S. X. Wei, C.-M. Lawrence Wu, W. Y. Guo, and L. M. Zhao, "Theoretical characterization of ruthenium complexes containing functionalized bithiophene ligands for dyesensitized solar cells," Journal of Organometallic Chemistry, vol. 696, no. 8, pp. 1632-1639, 2011.

[26] X. Q. Lu, C.-M. Lawrence Wu, S. X. Wei, and W. Y. Guo, "DFT/TD-DFT investigation of electronic structures and spectra properties of Cu-based dye sensitizers," The Journal of Physical Chemistry A, vol. 114, no. 2, pp. 1178-1184, 2010.

[27] X. Q. Lu, S. X. Wei, C.-M. Lawrence Wu, S. R. Li, and W. Y. Guo, "Can polypyridyl $\mathrm{Cu}(\mathrm{I})$-based complexes provide promising sensitizers for dye-sensitized solar cells? A theoretical insight into $\mathrm{Cu}(\mathrm{I})$ versus $\mathrm{Ru}(\mathrm{II})$ sensitizers," The Journal of Physical Chemistry C, vol. 115, no. 9, pp. 3753-3761, 2011.

[28] X. Q. Lu, S. X. Wei, C.-M. Lawrence Wu et al., "Theoretical insight into the spectral characteristics of Fe(II)-based complexes for dye-sensitized solar cells-Part I: Polypyridyl ancillary ligands," International Journal of Photoenergy, vol. 2011, Article ID 316952, 11 pages, 2011.

[29] M. J. Frisch, G. W. Trucks, H. B. Schlegel et al., Gaussian 09, Revision A.1, Gaussian, Wallingford, Conn, USA, 2009.

[30] C. Angeli, M. Pastore, and R. Cimiraglia, "New perspectives in multireference perturbation theory: the n-electron valence state approach," Theoretical Chemistry Accounts, vol. 117, no. 5-6, pp. 743-754, 2007.

[31] M. Cossi and V. Barone, "Time-dependent density functional theory for molecules in liquid solutions," The Journal of Chemical Physics, vol. 115, no. 10, pp. 4708-4717, 2001.

[32] S. Miertš, E. Scrocco, and J. Tomasi, "Electrostatic interaction of a solute with a continuum. A direct utilizaion of $A B$ initio molecular potentials for the prevision of solvent effects," Chemical Physics, vol. 55, no. 1, pp. 117-129, 1981.

[33] M. Cossi, V. Barone, R. Cammi, and J. Tomasi, "Ab initio study of solvated molecules: a new implementation of the polarizable continuum model," Chemical Physics Letters, vol. 255, no. 4-6, pp. 327-335, 1996.

[34] V. Barone and M. Cossi, "Quantum calculation of molecular energies and energy gradients in solution by a conductor solvent model," The Journal of Physical Chemistry A, vol. 102, no. 11, pp. 1995-2001, 1998.

[35] F. D. Angelis, S. Fantacci, and A. Selloni, "Alignment of the dye's molecular levels with the $\mathrm{TiO}_{2}$ band edges in dye-sensitized solar cells: a DFT-TDDFT study," Nanotechnology, vol. 19, no. 42, Article ID 424002, 2008.

[36] J. Feng, Y. Jiao, W. Ma, M. K. Nazeeruddin, M. Grätzel, and S. Meng, "First principles design of dye molecules with ullazine donor for dye sensitized solar cells," The Journal of Physical Chemistry C, vol. 117, no. 8, pp. 3772-3778, 2013.

[37] W. J. Fan, D. Z. Tan, and W.-Q. Deng, "Acene-modified triphenylamine dyes for dye-sensitized solar cells: a computational study," ChemPhysChem, vol. 13, no. 8, pp. 2051-2060, 2012.

[38] K. P. Guo, K. Y. Yan, X. Q. Lu et al., "Dithiafulvenyl unit as a new donor for high-efficiency dye-sensitized solar cells: synthesis and demonstration of a family of metal-free organic sensitizers," Organic Letters, vol. 14, no. 9, pp. 2214-2217, 2012.

[39] G. L. Zhang, H. Bala, Y. M. Cheng et al., "High efficiency and stable dye-sensitized solar cells with an organic chromophore featuring a binary $\pi$-conjugated spacer," Chemical Communications, no. 16, pp. 2198-2200, 2009.

[40] M. Pastore, E. Mosconi, F. de Angelis, and M. Grätzel, "A computational investigation of organic dyes for dye-sensitized solar cells: benchmark, strategies, and open issues," The Journal of Physical Chemistry C, vol. 114, no. 15, pp. 7205-7212, 2010.

[41] J. Preat, C. Michaux, D. Jacquemin, and E. A. Perpète, "Enhanced efficiency of organic dye-sensitized solar cells: triphenylamine derivatives," The Journal of Physical Chemistry $C$, vol. 113, no. 38, pp. 16821-16833, 2009.

[42] J. Preat, C. Michaux, D. Jacquemin, and E. A. Perpète, "Enhanced efficiency of organic dye-sensitized solar cells: triphenylamine derivatives," The Journal of Physical Chemistry $C$, vol. 113, no. 38, pp. 16821-16833, 2009.

[43] M. Pastore, E. Mosconi, F. de Angelis, and M. Grätzel, "A computational investigation of organic dyes for dye-sensitized solar cells: benchmark, strategies, and open issues," The Journal of Physical Chemistry C, vol. 114, no. 15, pp. 7205-7212, 2010.

[44] N. Santhanamoorthi, C.-M. Lo, and J.-C. Jiang, "Molecular design of porphyrins for dye-sensitized solar cells: a DFT/TDDFT study," The Journal of Physical Chemistry Letters, vol. 4, no. 3, pp. 524-530, 2013.

[45] C.-Y. Chen, M. Wang, J.-Y. Li et al., "Highly efficient lightharvesting ruthenium sensitizer for thin-film dye-sensitized solar cells," ACS Nano, vol. 3, no. 10, pp. 3103-3109, 2009.

[46] H. S. Nalwa, Handbook of Advanced Electronic and Photonic Materials and Devices: Conducting Polymers, Academic Press, San Diego, Calif, USA, 2001. 

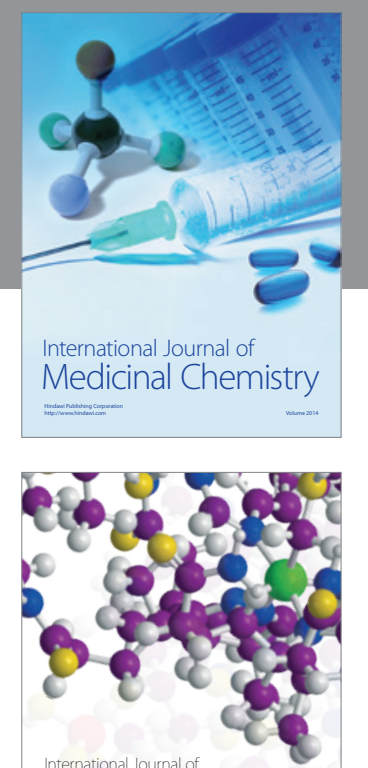

\section{Carbohydrate} Chemistry

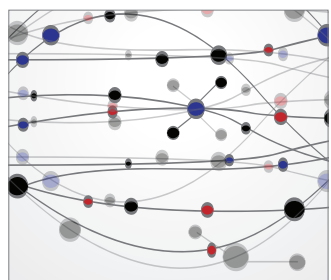

The Scientific World Journal
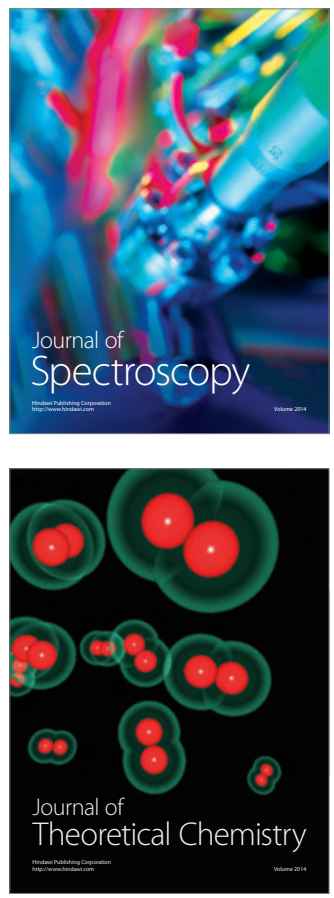
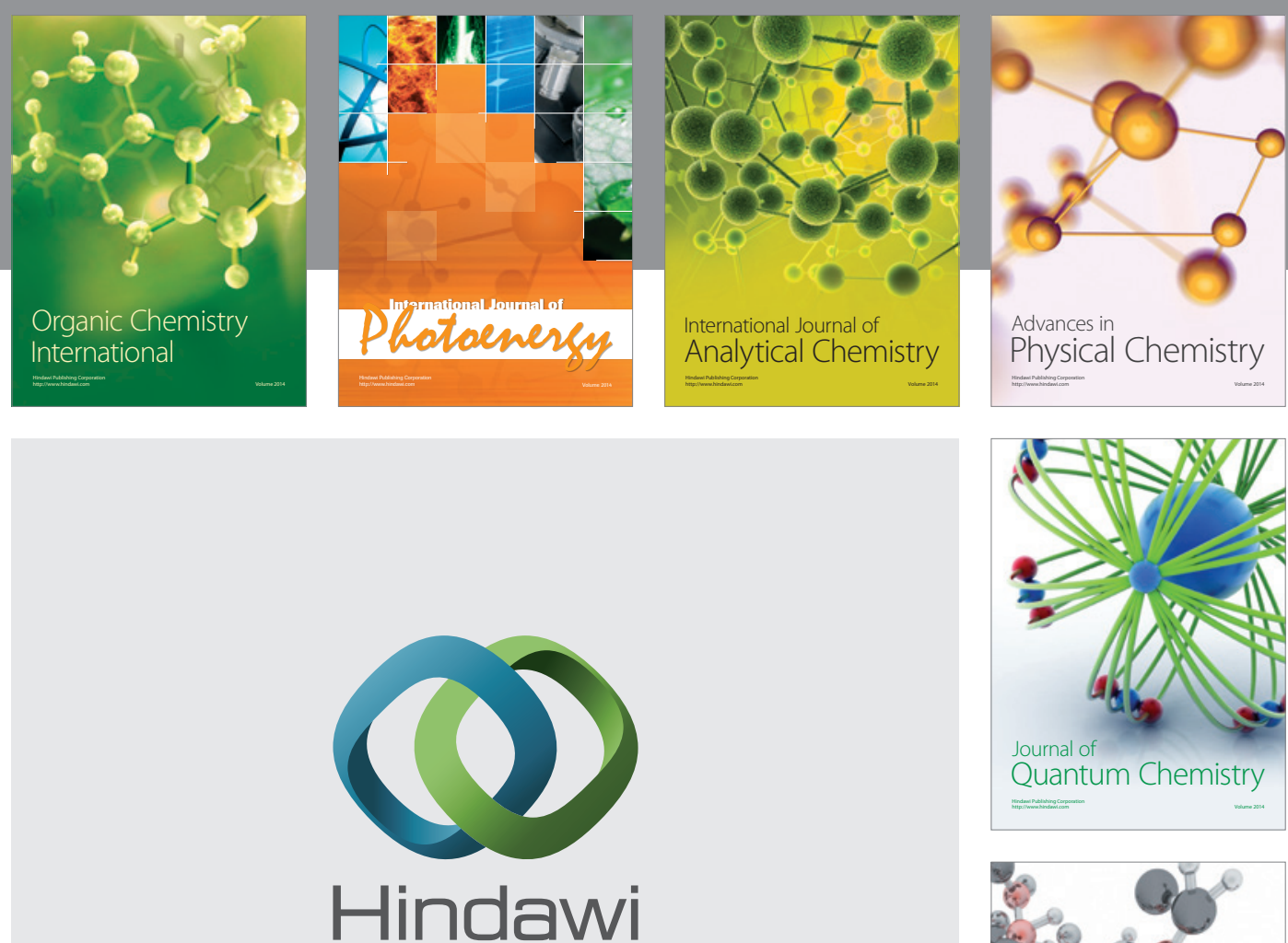

Submit your manuscripts at

http://www.hindawi.com

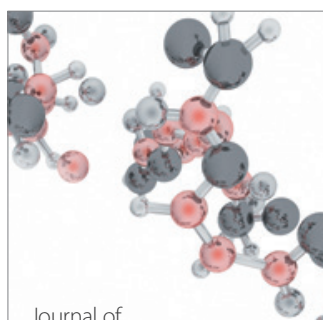

Analytical Methods

in Chemistry

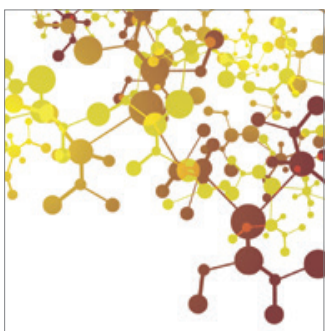

Journal of

Applied Chemistry

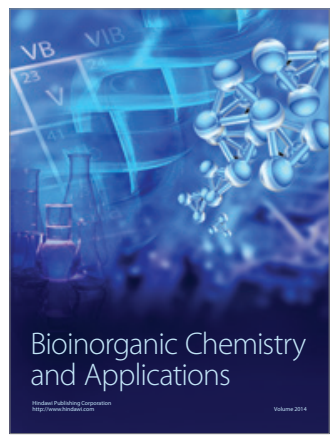

Inorganic Chemistry
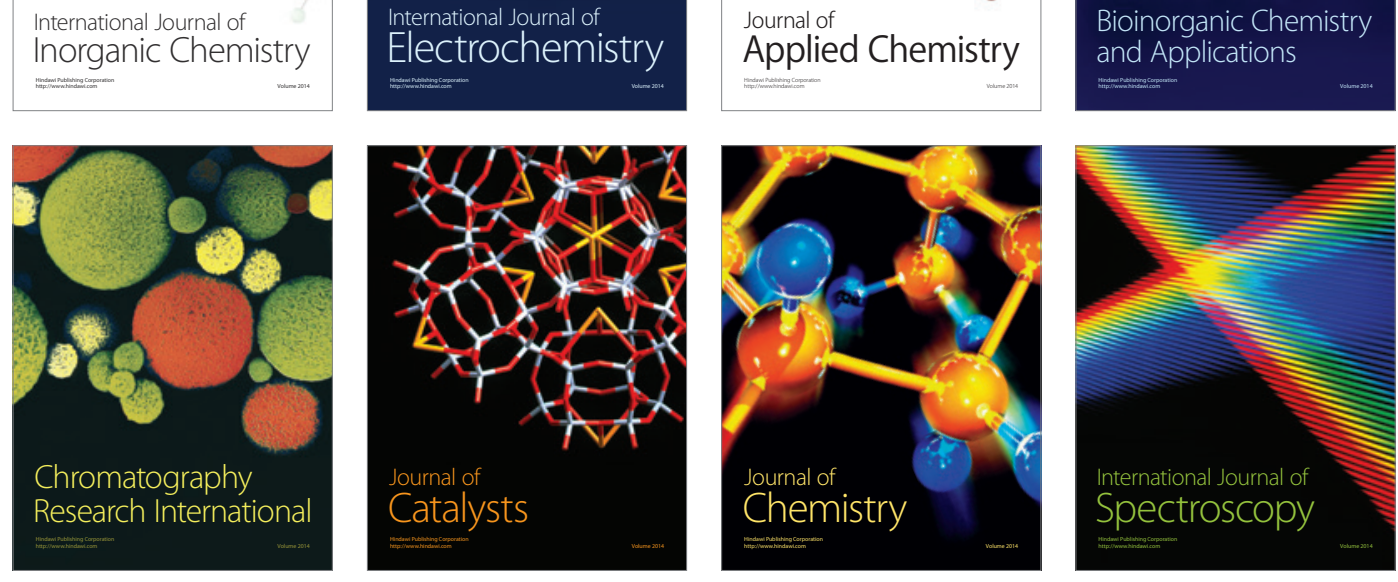